\title{
High glucose promotes apoptosis and autophagy of MC3T3-E1 osteoblasts
}

\author{
Pei Zhang, Jing Liao, Xiaoju Wang, Zhengping Feng
}

\begin{abstract}
Department of Endocrinology, First Affiliated Hospital of Chongqing Medical University, Chongqing, China
\end{abstract}

Submitted: 1 August 2019; Accepted: 7 March 2020

Online publication: 29 November 2020

Arch Med Sci 2023; 19 (1): 138-150

DOI: https://doi.org/10.5114/aoms.2020.101307

Copyright @ 2020 Termedia \& Banach

\section{Abstract}

Introduction: Diabetes and osteoporosis are common metabolic diseases. Abnormal high glucose can lead to the apoptosis of osteoblasts. Autophagy is a highly conserved cellular process that degrades proteins or organelles. In the present study, we comparatively analyzed the effects of high glucose and glucose fluctuation on apoptosis and autophagy of MC3T3-E1 osteoblasts.

Material and methods: MC3T3-E1 cells were respectively treated with different concentrations of D-glucose: $5.5 \mathrm{mM}$ for the control group, $25 \mathrm{mM}$ for the high glucose group and 5.5/25 mM for the glucose fluctuation group. Results: High glucose and glucose fluctuation decreased MC3T3-E1 proliferation and activated autophagy. Also, high glucose and glucose fluctuation might induce the production of reactive oxygen species, decline the mitochondrial membrane potential and trigger apoptosis. The differences in the glucose fluctuation treatment group were more significant. Moreover, $\mathrm{N}$-acetylcysteine, an antioxidant reagent, dramatically eliminated the intracellular reactive oxygen species induced by high glucose and glucose fluctuation, and significantly inhibited the autophagy and apoptosis in MC3T3-E1 osteoblasts. Furthermore, treatment with chloroquine, an inhibitor of autophagy, significantly increased the apoptosis of MC3T3-E1 osteoblasts.

Conclusions: High glucose, especially high glucose fluctuation, inhibits proliferation and promotes apoptosis and autophagy of MC3T3-E1 osteoblasts. This may occur through inducing oxidative stress and mitochondrial damage in the osteoblasts.

Key words: autophagy, oxidative stress, apoptosis, osteoblast, glucose fluctuation.

\section{Introduction}

Diabetes and osteoporosis are common chronic metabolic diseases worldwide [1-4]. Diabetes may be an important reason for osteoporotic fractures. Type 1 diabetes mellitus is known to damage bone microstructure [5]. Additionally, type 2 diabetes mellitus can damage bone microstructure by compromising matrix structure and cell function, which induce increased osteoblast apoptosis, decreased osteoblast differentiation, and increased bone resorption [6]. Clinically, persistent high glucose and glucose fluctuation are two manifestations of patients with hyperglycemia. However, the specific molecular mechanism underlying the effects of hyperglycemia on osteoporosis remains unclear [7].

\author{
Corresponding author: \\ Zhengping Feng \\ Department of Endocrinology \\ First Affiliated Hospital \\ of Chongqing Medical \\ University \\ No. 1 Youyi Road \\ Yuzhong District, Chongqing \\ 400016, China \\ Phone: +86-13618329135 \\ E-mail: fengzhengping@sina. \\ com
}


Autophagy, an important pathway for degrading the damaged organelles and substances in cells, is a highly conserved cytoprotective process in which cytoplasmic contents are sequestered, transported to lysosomes by double-membrane autophagosomes and degraded [8]. It is also an adaptive response to help cells to survive various stresses, such as starvation, oxidative stress, hypoxia or infection, or even fatal stress $[9,10]$. Nevertheless, excessive autophagy may result in excessive self-digestion or even death [11]. Autophagy has been reported to play an important role in the normal physiological function of bone cells. For example, Song et al. reported that autophagy activation can induce a survival response to protect bone marrow-derived mesenchymal stem cells from oxidative stress [12]. Meanwhile, autophagy is a double-edged sword. Several studies have demonstrated autophagy deficiency may lead to bone cell dysfunction. For instance, autophagy deficiency could cause declined mineralization capacity in osteoblasts, reduced cellular projections in osteocytes, and retention of organelles in osteocytes [1315]. Among the markers of autophagy, Beclin 1 is an essential protein that signals the onset of autophagy [16]. LC3-II is the only protein marker that is reliably associated with completed autophagosomes, which is typically characterized as an autophagosome marker [17]. In addition to LC3-II, P62 is also used as a protein marker of autophagic degradation [18].

Osteoblasts, the main functional cells for bone formation, play a crucial role in the maintenance of bone homeostasis. Recent studies have shown that high glucose can promote the production of reactive oxygen species (ROS), increase the oxidative stress, and impair the mitochondrial function, which lead to various complications of diabetes [19-21]. Meanwhile, production of ROS could induce apoptosis in various types of cells, such as pancreatic beta cells, endothelial cells, and osteoblasts [22-24]. ROS might affect the functions of osteoblasts, which has been confirmed by our group [25]. Additionally, Li et al. found a close correlation among ROS, autophagy and apoptosis [26]. Accumulation of ROS leads to oxidative damage, which results in mitochondrial dysfunction and cell damage [27, 28]. Meanwhile, apoptosis and autophagy both can be induced by various stress states via ROS generation [29, 30]. Although apoptosis and autophagy are two distinct processes that present clearly different biochemical and morphological features, there is a close relationship and regulatory network between them [31]. However, no previous study has investigated the role of autophagy in the hyperglycemia-induced apoptosis in osteoblasts.

Herein, we investigated the effects of high glucose (continuous high glucose and glucose fluctuation) on autophagy and apoptosis of MC3T3-E1 osteoblasts. The underlying mechanisms were further analyzed and discussed.

\section{Material and methods}

\section{Cell culture and treatment}

MC3T3-E1 osteoblasts were obtained from the Chinese Center for Type Culture Collection (Shanghai, China). Cells were cultured in $\alpha$-Minimum Essential Medium ( $\alpha$-MEM, HyClone, USA) supplemented with $10 \%$ fetal bovine serum (PAN Biotech, Aidenbach, Germany), 1\% sodium pyruvate (Solarbio, Beijing, China) and 1\% penicillin/streptomycin (Beyotime, Shanghai, China) in a humidified incubator with $5 \% \mathrm{CO}_{2}$ at $37^{\circ} \mathrm{C}$. Cells were treated with different concentrations of D-glucose (Sigma, St. Louis, MO, USA): $5.5 \mathrm{mM}$ for the control group (Ctr group), $25 \mathrm{mM}$ for the high glucose group (HG group) and $5.5 / 25 \mathrm{mM}$ for the glucose fluctuation group (GF group). N-acetylcysteine (NAC, $2 \mathrm{mM}$, Beyotime, Shanghai, China) and chloroquine (CQ, $10 \mu \mathrm{M}$, Sigma, St Louis, MO, USA) were used as an antioxidant and autophagy inhibitor, respectively. The medium, containing different concentrations of D-glucose and $\mathrm{NAC} / \mathrm{CQ}$, in each group was changed every eight hours. All the treatment in this study lasted for $48 \mathrm{~h}$.

\section{Cell Counting Kit-8 assay (CCK-8)}

The CCK-8 kit (Dojindo, Kumamoto, Japan) is a highly sensitive, non-radioactive colorimetric method for determining the number of living cells in a cell proliferation experiment. The amount of formazan produced, which can be detected by a microplate reader, is directly proportional to the number of living cells. Briefly, MC3T3-E1 cells were seeded in 96-well plates at a density of $5 \times 10^{3}$ per well, and each sample was repeated six times. After incubation for $48 \mathrm{~h}, 10 \mu \mathrm{l}$ of CCK-8 solution was added to each well and incubated for another 1-4 h. Then, the optical density (OD) at $450 \mathrm{~nm}$ was measured by the microplate reader.

\section{Flow cytometry}

Apoptosis was measured with an Annexin V-FITC/PI Apoptosis Detection Kit (Tianjin Sungene Biotech, China). Cells were collected and suspended with $400 \mu \mathrm{l} 1 \times$ binding buffer. Then, $5 \mu \mathrm{l}$ of Annexin V-FITC was added to the cell suspension and incubated at $2-8^{\circ} \mathrm{C}$ for $15 \mathrm{~min}$. After that, $10 \mu \mathrm{l}$ of PI was added and incubated for $5 \mathrm{~min}$ at $2-8^{\circ} \mathrm{C}$ in the dark. Finally, cells were analyzed by flow cytometry (BD FACS Vantage SE). Annexin V-positive/PI-positive cells were considered as late apoptotic or necrotic cells.

\section{Measurement of ROS and MitoSOX}

Intracellular and mitochondrial ROS were detected by the 2', 7'-dichlorofluorescein diacetate (DCFH-DA) fluorescent probe (Beyotime, China) and MitoSOX Red (Invitrogen, USA), a mitochondrial su- 
peroxide indicator, respectively. Cells were plated at a density of $10^{4} /$ well in a 12 -well plate. After incubation for $24 \mathrm{~h}$, cells were respectively treated with different concentrations of glucose with or without $\mathrm{NAC}$ for another $48 \mathrm{~h}$.

According to the instructions for the DCFH-DA fluorescent probe, cells were first washed with basal medium and then incubated with a diluted DCFHDA probe solution $(1: 1000)$. During 30 min incubation, the plate was gently shaken every $5-10 \mathrm{~min}$ three times. Later, the cells were washed with basal medium again and the green fluorescence intensity was observed by inverted fluorescence microscope (ZEISS, Germany).

For analysis of the mitochondrial ROS, cells were seeded on a glass coverslip in 12-well plates and treated with different concentrations of glucose. After washing with PBS, cells were loaded with $0.5-1.0 \mathrm{ml}$ of MitoSOX $(5 \mu \mathrm{M})$ solution for $10 \mathrm{~min}$ at $37^{\circ} \mathrm{C}$ and then washed with buffer three times. MitoTracker was used to localize the mitochondria and its fluorescence intensity was observed by fluorescence microscope (ZEISS, Germany).

\section{JC-1 staining analysis}

To detect the mitochondrial transmembrane potential $(\Delta \Psi \mathrm{m})$ of MC3T3-E1 osteoblasts in each group, JC-1 staining was performed. Cells were seeded on the slides in a 12-well plate. After $48 \mathrm{~h}$ of treatment, the culture medium was discarded, and the cells were washed with PBS. Then, $1 \mathrm{ml}$ of fresh medium and $1 \mathrm{ml}$ of JC-1 staining working solution were added to each well, and the plate was incubated at $37^{\circ} \mathrm{C}$ for $20 \mathrm{~min}$. Later, the plate was washed twice with pre-chilled JC-1 staining buffer $(1 \times)$ and $2 \mathrm{ml}$ of fresh medium was added to each well. The fluorescence microscope (ZEISS, Germany) was used to observe the red and green fluorescence. Carbonyl cyanide 3-chlorophenylhydrazone (CCCP) was used as a positive control.

\section{Western blot}

Osteoblasts were lysed in RIPA lysis buffer with protease inhibitors (Beyotime, Shanghai, China). Proteins were collected after centrifugation. The protein concentrations were determined by BCA Protein Assay Kit (Beyotime, China). The protein samples were subjected to SDS-PAGE (8-12\%), and then transferred onto PVDF membranes (Millipore, Germany). After blocking with 5\% skimmed milk (Bio-Rad, USA) for at least $1 \mathrm{~h}$, the membranes were incubated with primary antibodies overnight at $4^{\circ} \mathrm{C}$, including rabbit anti-LC3 antibody (1: 1000, Novus), rabbit anti-Bnip3 antibody (1:2000, Abcam), rabbit anti-P62 antibody (1:1000, Abcam), rabbit anti-Beclin1antibody (1:1000, Abcam), mouse anti-caspase 3 antibody (1:500, Santa Cruz), rabbit anti-cleaved caspase 3 antibody $(1: 1000$, CST), rabbit anti-Bcl-2 antibody (1: 1000, CST), rabbit antiBax antibody (1:1000, CST) and mouse anti- $\beta$ actin antibody (1: 8000, Tianjin Sungene Biotech). Depending on the origin of the primary antibodies, the secondary antibody $(1: 7000-1: 10000)$ was added and incubated for $1 \mathrm{~h}$ at room temperature. The intensities of bands were quantified with $\mathrm{Fu}$ sion software (VILBER LOURMAT, Germany). All the results were normalized to $\beta$-actin.

\section{Immunofluorescence assay}

MC3T3-E1 osteoblasts were seeded on pre-coated glass coverslips and treated with different glucose concentrations. Then the coverslips were washed with PBS, fixed in 4\% paraformaldehyde for $30 \mathrm{~min}$, permeabilized with $0.25 \%$ Triton for $15 \mathrm{~min}$, and blocked with $5 \%$ bovine serum albumin for $1 \mathrm{~h}$ at room temperature. The cells were incubated with primary antibodies against LC3 (1 : 200) and P62 (1 : 200) overnight at $4^{\circ} \mathrm{C}$, followed by incubation with the matching biotinylated secondary antibody for $1 \mathrm{~h}$ in the dark at room temperature. Then, nucleus was stained with 4,6-diamidino-2-phenylindole (DAPI) for $5 \mathrm{~min}$, and the fluorescence intensity was observed under the fluorescence microscope.

\section{Transmission electron microscopy (TEM) analysis}

Cells (at least 10\%/group) were collected and fixed with glutaraldehyde. The samples were observed and photographed using TEM $(\mathrm{H}-7500$, Hitachi Company, Japan). Autophagic vacuoles were counted from four randomly selected fields, and the autophagic vacuoles per field were presented as mean \pm SEM.

\section{Statistical analysis}

Data were analyzed using GraphPad Prism Version7.0a software (GraphPad Software, San Diego, CA, USA). Multiple group analysis was performed by one-way ANOVA or two-way ANOVA. Independent experiments were performed three times. The data were expressed as mean \pm SEM. The value of $p$ $<0.05$ was considered statistically significant.

\section{Results}

High glucose and glucose fluctuation decrease proliferation and induce apoptosis in osteoblasts

To test the effects of high glucose and glucose fluctuation on the apoptosis and proliferation of osteoblasts, flow cytometry and CCK-8 assay, respectively, were performed. The late apoptotic rates of the HG group and the GF group were 2.3and 3.7-fold those of the control group, respec- 
tively ( $p<0.01$, Figures 1 A and B) and the proliferation rates of the $\mathrm{HG}$ group and the GF group were significantly lower, especially the GF group ( $p<0.01$, Figure $1 \mathrm{C}$ ). Compared with the HG group, the GF group had a significantly higher apoptosis rate $(p<0.01$, Figure 1 B) but a significantly lower OD value, which indicates a lower cell proliferation rate $(p<0.05$, Figure $1 \mathrm{C})$. These results indicate that high glucose and glucose fluctuation could inhibit proliferation and induce apoptosis in the osteoblasts.

\section{High glucose and glucose fluctuation induce oxidative stress and mitochondrial damage in osteoblasts}

To assess the effect of high glucose and glucose fluctuation on the ROS production in the osteoblasts, the ROS-sensitive fluorescent probe DCFHDA and MitoSOX were used. There was an increase of fluorescence intensity in the HG and GF groups, especially in the latter (Figure $2 \mathrm{~A}$ ). Thus, high glucose and glucose fluctuation might promote the intracellular and mitochondrial ROS production.

As reported by Pérez et al., intracellular mitochondrial damage was usually accompanied by a decrease of $\Delta \Psi \mathrm{m}$ [32], which could be monitored by the fluorescent probe JC-1. In the control group, there were a lot of JC-1 aggregates shown as red fluorescence, and a small number of JC-1 monomers shown as green fluorescence, whereas there were more JC-1 monomers and fewer JC-1 aggregates in the HG and GF groups, which were similar to the CCCP group (positive control) (Figure 2 B). The green fluorescence intensity in the GF group was dramatically lower than in the control group and the HG group, indicating that high glucose and glucose fluctuation could cause $\Delta \Psi \mathrm{m}$ reduction and mitochondrial damage in the osteoblasts.

\section{High glucose and glucose fluctuation induce autophagy in osteoblasts}

To investigate whether the high glucose and glucose fluctuation may induce autophagy in the osteoblasts, we performed TEM, western blot, and immunofluorescence, respectively. Autophagosomes are autophagy vesicles with an acidic double-membrane structure [33]. The TEM results showed more autophagosomes in the GF group than in the HG group and the control group (Figure $3 \mathrm{~A}$ ). The protein expression of LC3-II and

\section{A}

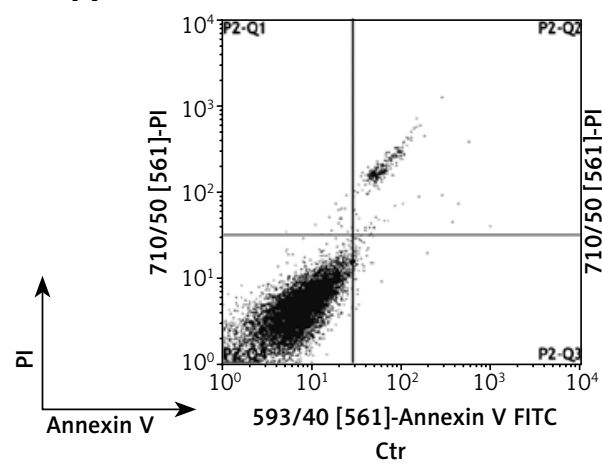

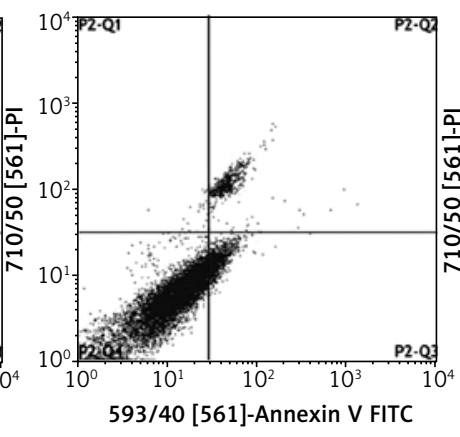

HG

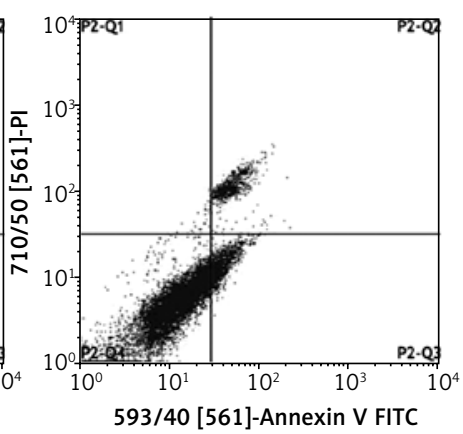

GF
B

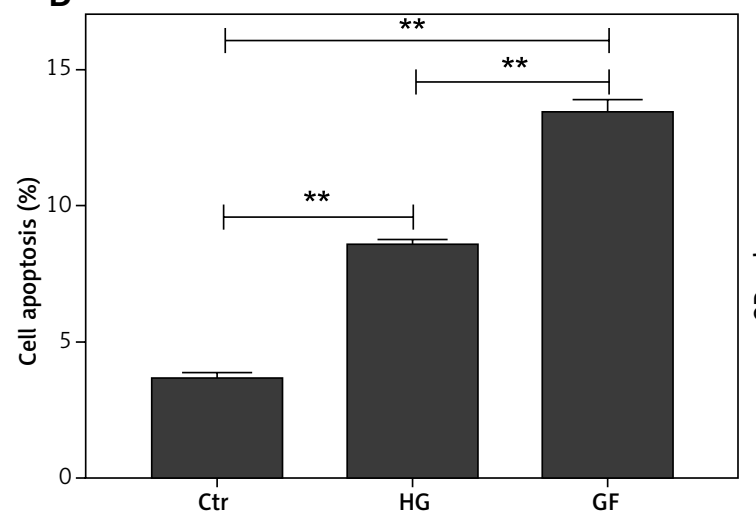

C

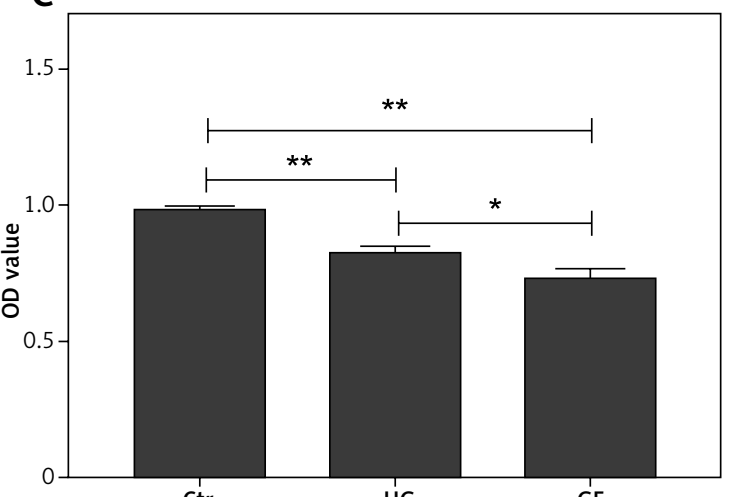

Figure 1. Effects of high glucose and glucose fluctuation on apoptosis and proliferation of osteoblasts. A - Cell apoptosis rate was analyzed by flow cytometry after Annexin V/PI staining. B - The late apoptotic rate was quantified and shown. C - CCK-8 assay was used to detect cell proliferation. OD value = optical density value. One-way ANOVA was used to analyze the data. Independent experiments were performed three times. Data are expressed as the mean $\pm \mathrm{SEM} ;{ }^{*} p<0.05,{ }^{* *} p<0.01$ 


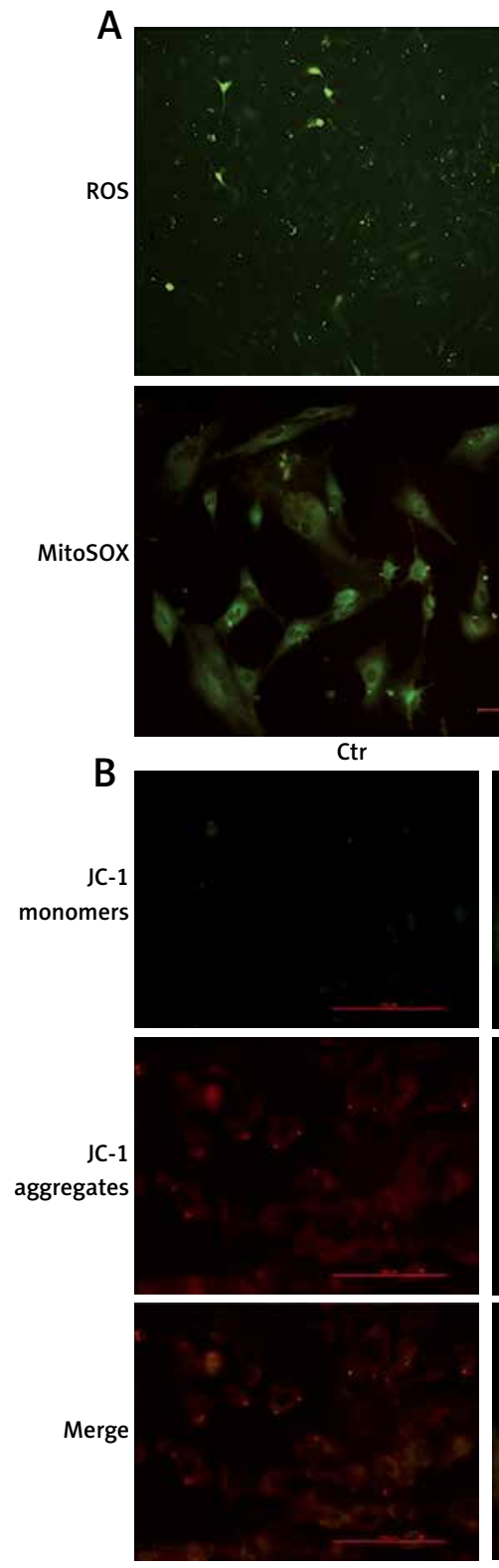

Ctr
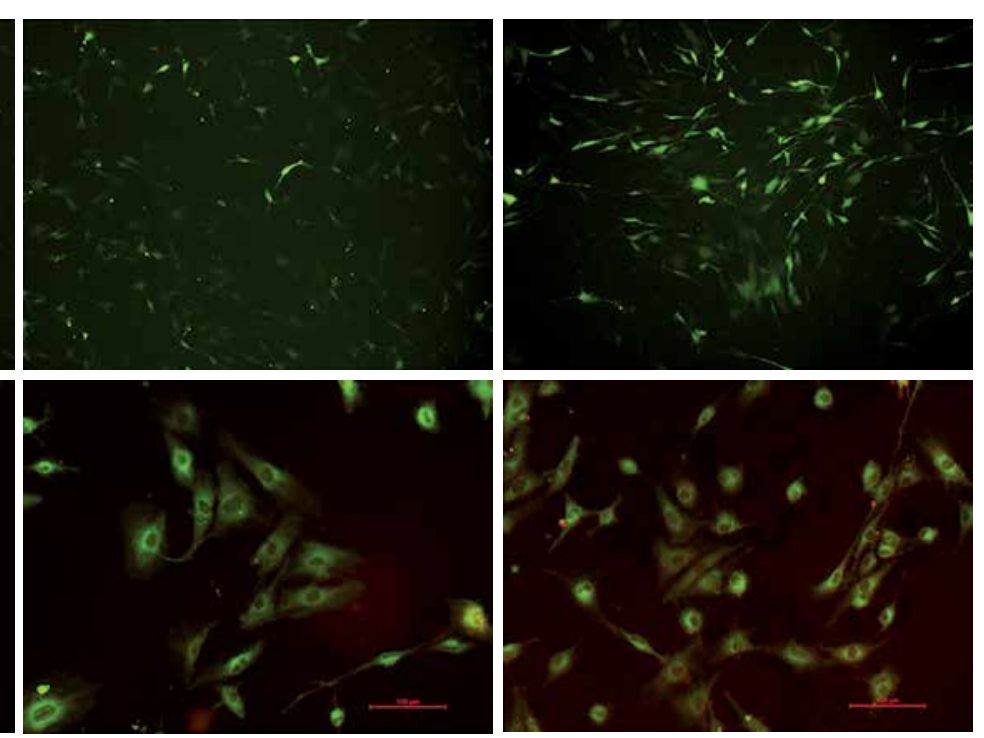

HG
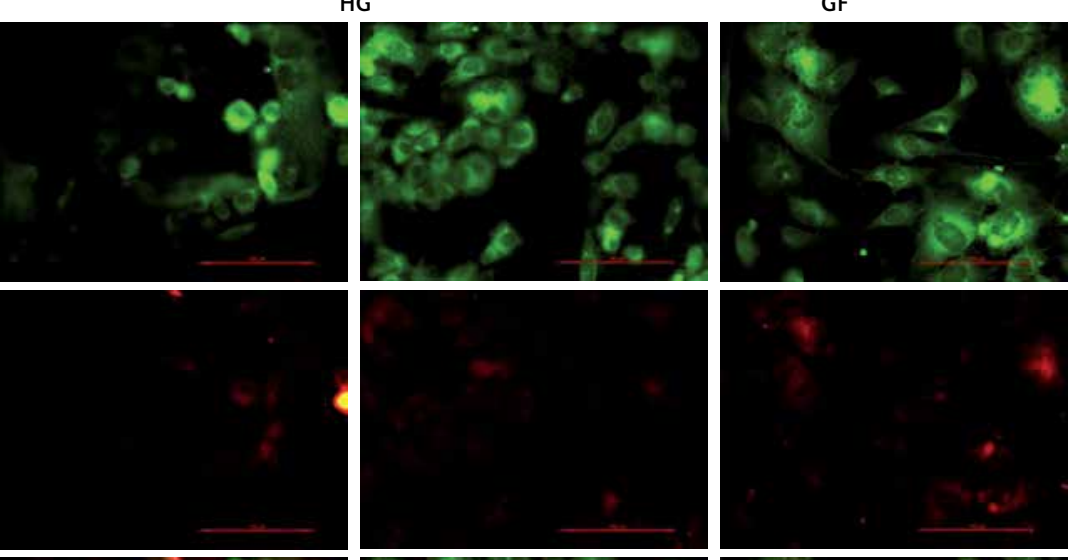

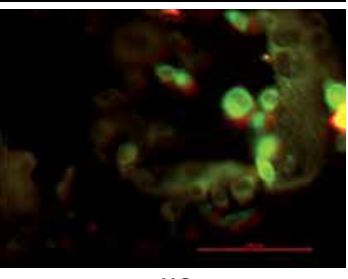

HG

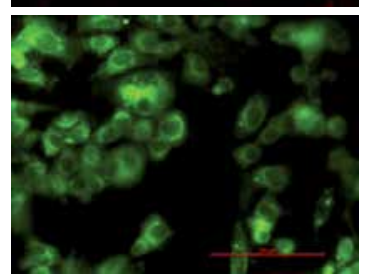

GF

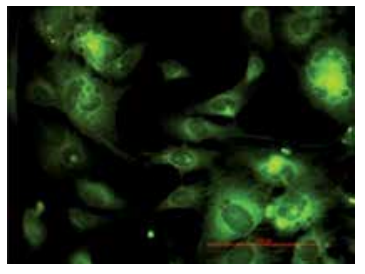

CCCP

Figure 2. Effects of high glucose and glucose fluctuation on ROS and $\Delta \Psi \mathrm{m}$ of osteoblasts. A - Fluorescence images of ROS detected by DCFH-DA probe (green), and immunofluorescence images of mitochondrial ROS detected by MitoSOX. In the lower panel, fluorescence of green and red was MitoTracker and MitoSOX in each group (200x; scale bar $=100 \mathrm{~mm})$. B $-\Delta \Psi \mathrm{m}$ was analyzed by JC-1 staining. Representative images are shown (200x; scale bar = $100 \mathrm{~mm})$. CCCP: Carbonyl cyanide 3-chlorophenylhydrazone

Beclin1 were increased in the osteoblasts, while the expression of p62 expression was apparently decreased, as determined by Western blot. Compared with high glucose treatment, the glucose fluctuation treatment could significantly increase the protein level of LC3-II and Beclin1, and decreased p62 expression (Figure 3 B). Immunofluorescence showed that LC3-II expression was increased, while p62 expression was decreased in $\mathrm{HG}$ and GF groups (Figure $3 \mathrm{C}$ ). These findings reveal that high glucose and glucose fluctuation, especially glucose fluctuation, may induce autophagy in the osteoblasts.

\section{Role of oxidative stress on autophagy in osteoblasts}

$\mathrm{N}$-acetylcysteine, an antioxidant reagent, is reported to decrease the production of ROS in the osteoblasts [25]. To determine ROS level after NAC treatment, a DCFH-DA probe was used. As shown in Figure $4 \mathrm{~A}$, the fluorescence intensity of ROS in the HG + NAC and GF + NAC groups was apparently lower than that in the HG and GF groups.

To test the role of oxidative stress on autophagy in the osteoblasts, cells were firstly incubated with NAC and then protein levels were detected by Western blot. Compared with the respective 

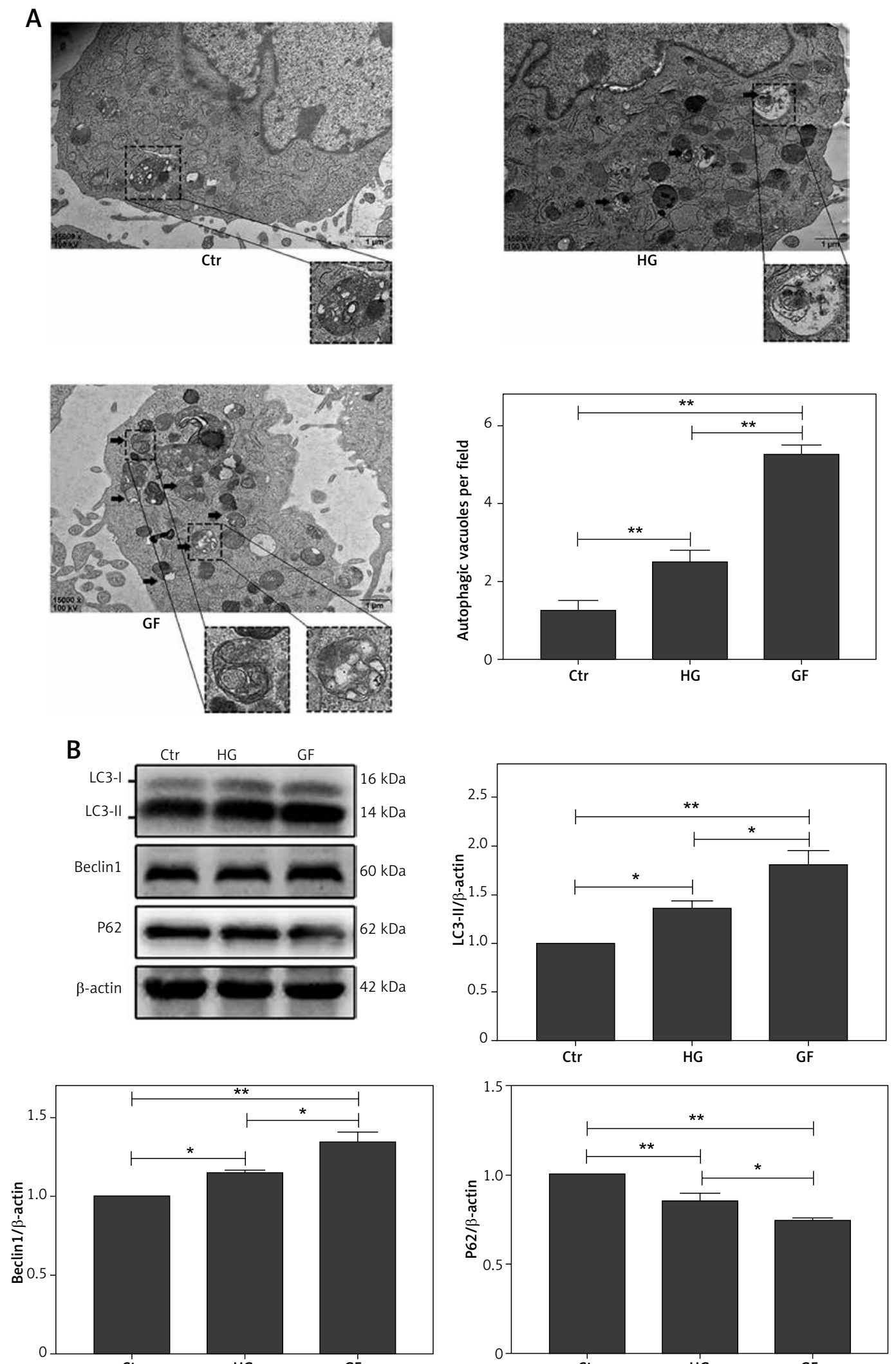

GF

Ctr

GF

Figure 3. Effect of high glucose and glucose fluctuation on autophagy in the osteoblasts. A - Representative transmission electron micrographs of the osteoblasts and data of autophagic vacuoles per field are shown (15000x, scale bar $=1 \mathrm{~mm}$ ). B - Western blot was used to analyze the expression of autophagy related proteins. Representative Western blot and quantitative analysis of the protein level of LC3, Beclin 1 and p62 in the osteoblasts after different treatments are shown. One-way ANOVA was used to analyze the data. Independent experiments were performed three times. Data are expressed as the mean $\pm \mathrm{SEM} ;{ }^{*} p<0.05,{ }^{* *} p<0.01$ 

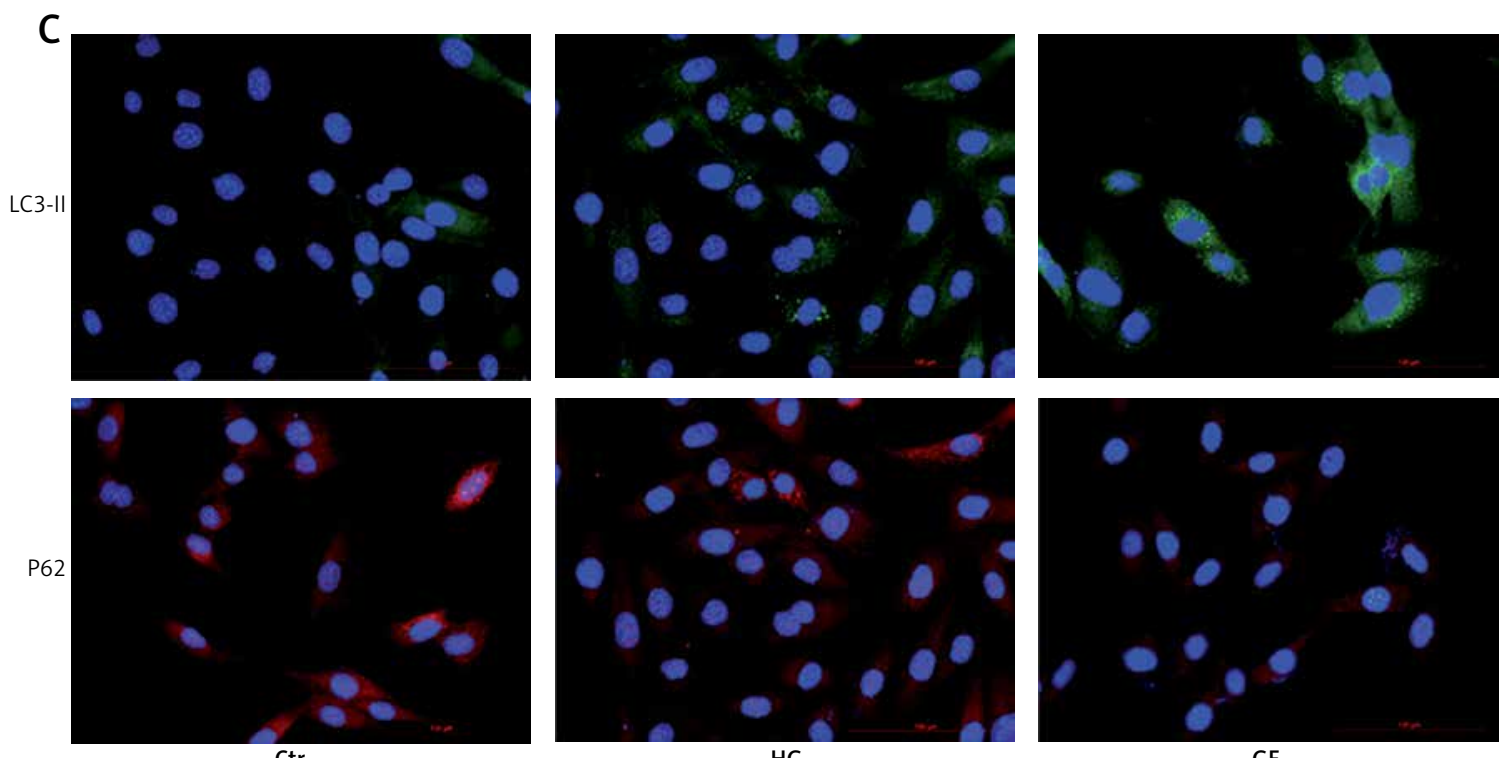

Figure 3. Cont. Effect of high glucose and glucose fluctuation on autophagy in the osteoblasts. C - Immunofluorescence assay $(200 \times$, scale bare $=100 \mathrm{~mm})$ was used to show the expression of LC3-II and p62. Fluorescence of blue, green and red was DAPI, LC3-II and p62, respectively

HG and GF group, NAC reduced the expression of LC3-II, Beclin1 and Bnip3 and increased p62 expression in the HG + NAC group and GF + NAC group (Figures $4 \mathrm{~B}$ and $\mathrm{C}$ ). In addition, the expression of $p$-Akt and p-mTOR in both HG and GF groups, especially in the GF group, was lower than in the control group, while NAC abolished this effect (Figures $4 \mathrm{~B}$ and $\mathrm{C}$ ). These results suggest that the autophagy induced by high glucose and glucose fluctuation is inhibited after the decrease of oxidative stress by NAC.

\section{Effects of oxidative stress and autophagy on apoptosis in osteoblasts}

We previously demonstrated that high glucose and glucose fluctuation could induce apoptosis, oxidative stress and autophagy, while NAC could attenuate oxidative stress and inhibit autophagy. To further determine the effects of oxidative stress and autophagy on the apoptosis of osteoblasts, we used flow cytometry to test the apoptosis of osteoblasts treated with high glucose or glucose fluctuation in the presence or absence of NAC and CQ. Compared with the HG and GF groups, NAC significantly decreased their apoptotic rates (Figures $5 \mathrm{~A}$ and $\mathrm{B}, p<0.01)$. Meanwhile, $\mathrm{Bcl}-2$ protein expression was lower in the HG and GF groups in comparison to the control group, while Bax, caspase 3 , cleaved caspase 3 and cytochrome $C$ expression levels were significantly increased (Figure $5 \mathrm{C}$ ). Compared with the HG group, the protein level of $\mathrm{Bcl}-2$ was much lower in the GF group, while the levels of Bax, caspase 3, cleaved caspase 3 and cytochrome $\mathrm{C}$ expression were much higher. However, NAC attenuated these effects (Figure $5 \mathrm{C}$ ).
After treatment with CQ (an autophagy inhibitor), LC3-II expression was significantly increased (Figures $6 \mathrm{~A}$ and $\mathrm{B}$ ), indicating that autophagy induced by high glucose or glucose fluctuation treatments was inhibited. Also, the apoptotic rates were significantly higher in CQ treatment groups $(\mathrm{HG}+\mathrm{CQ}$ and $\mathrm{GF}+\mathrm{CQ})$ than in the absence of CQ (Figures $6 \mathrm{C}$ and $\mathrm{D}$ ).

The above results indicated that inhibition of oxidative stress could decrease apoptosis, while inhibition of autophagy could increase apoptosis in osteoblasts.

\section{Discussion}

Diabetes and osteoporosis are two common chronic metabolic diseases. Previous studies have shown that diabetes could increase the risk of osteoporotic fracture [34, 35]. The osteoblasts and osteoclasts are known to play extremely important roles in the reconstruction of bone. The maintenance of bone homeostasis depends on the stable function of osteoblasts and osteoclasts [36]. High glucose leads to abnormal bone metabolism, osteoblast dysfunction and bone formation decrease [37]. However, the molecular mechanism of abnormal bone metabolism in diabetes has not been well studied.

Recent studies have shown that glucose fluctuation plays an important role in various chronic complications induced by diabetes [38-44]. In patients with type 1 and type 2 diabetes mellitus, glucose fluctuation has been reported to be closely related to oxidative stress [38, 45]. Moreover, in comparison to high glucose, there was a stronger correlation between glucose fluctuation and oxi- 


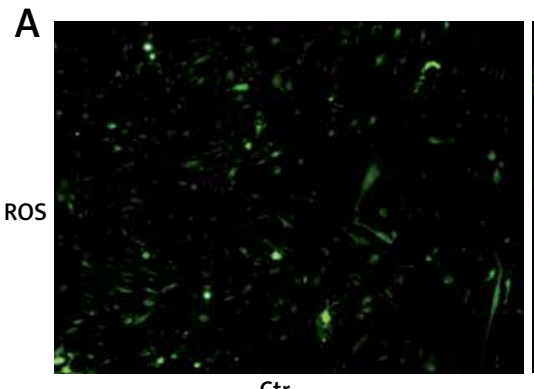

Ctr

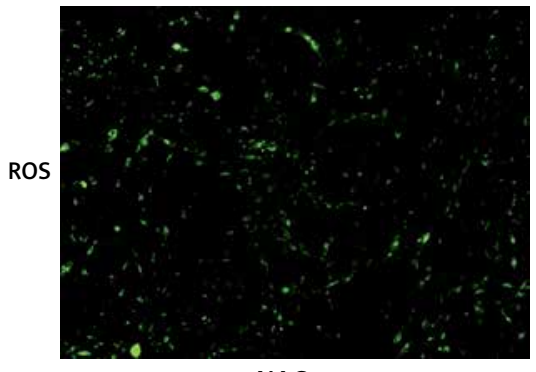

NAC

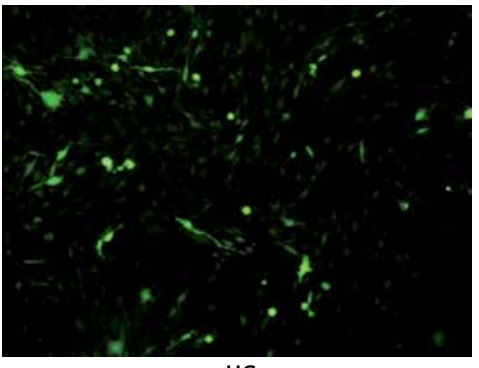

HG

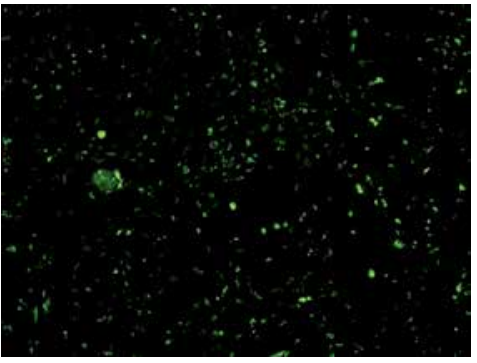

$\mathrm{HG}+\mathrm{NAC}$
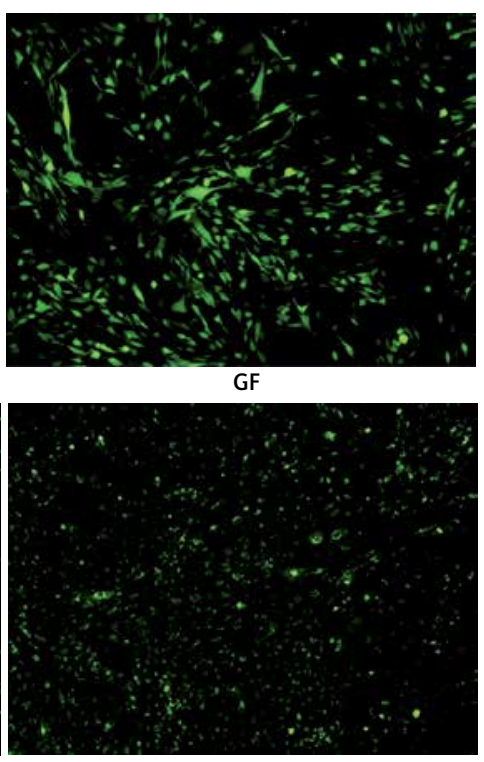

$\mathrm{GF}+\mathrm{NAC}$

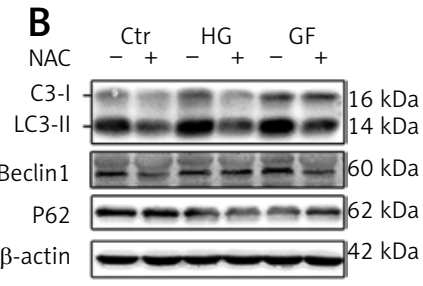

C
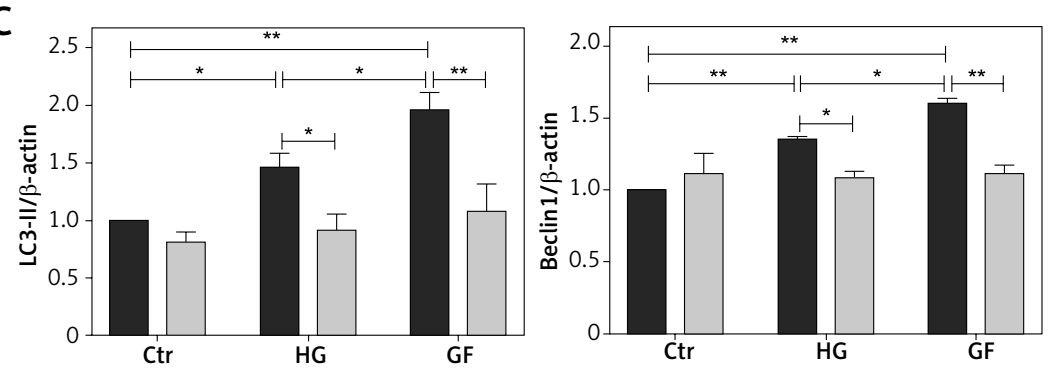

$\mathrm{NAC}-\mathrm{Ctr}_{+}{ }_{-}^{\mathrm{HG}}{ }_{-} \mathrm{GF}_{+}$
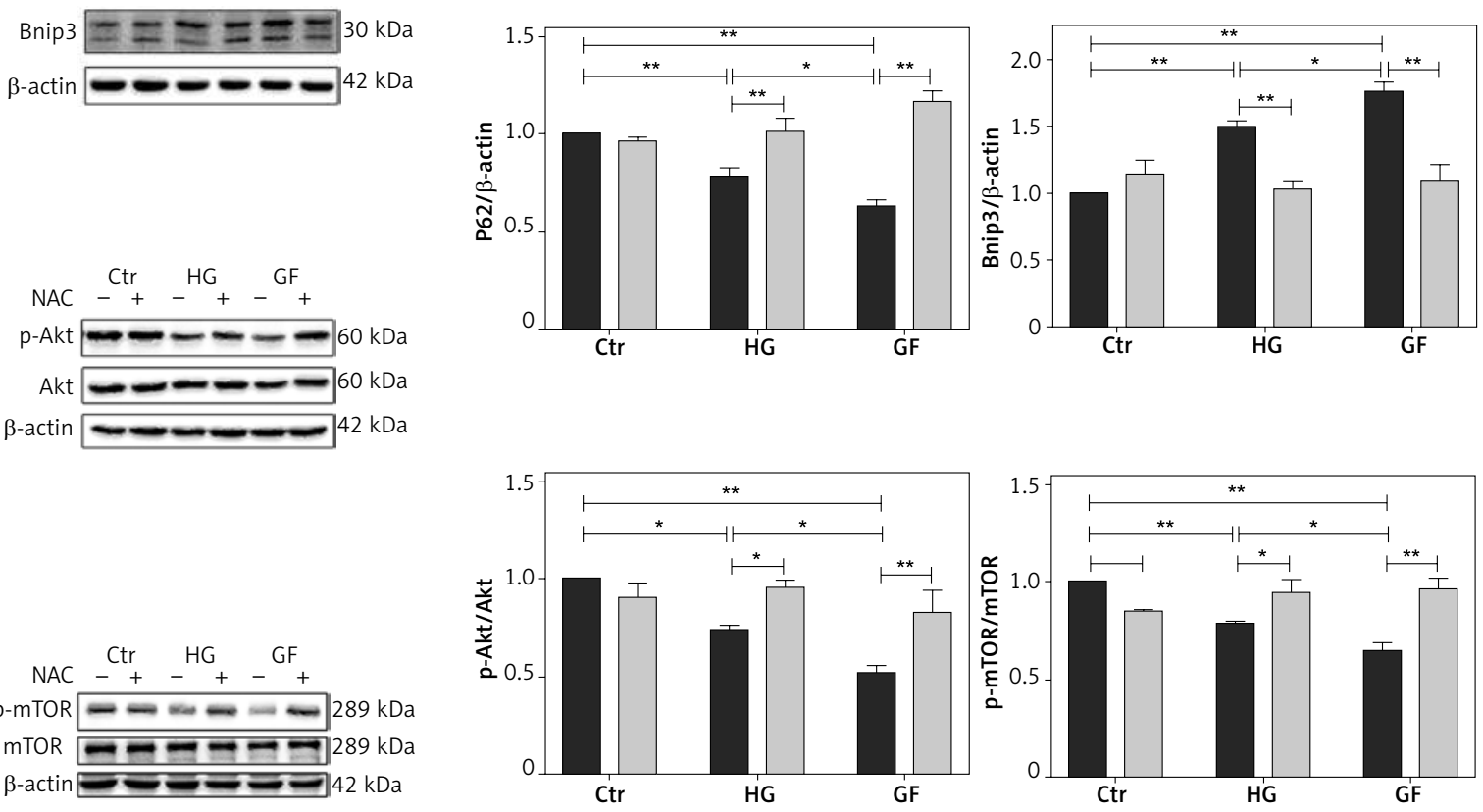

Figure 4. Effects of NAC on ROS production and autophagy-related proteins. A - Representative images of ROS detected by DCFH-DA probe (200x). B - Representative Western blot and C - quantitative analysis of LC3-II, Beclin1, p62, Bnip3, p-Akt/Akt and p-mTOR/mTOR induced by high glucose and glucose fluctuation w/wo the presence of NAC. Two-way ANOVA was used to analyze the data. Independent experiments were performed three times. Data are expressed as the mean $\pm \mathrm{SEM} ;{ }^{*} p<0.05,{ }^{* *} p<0.01$ 

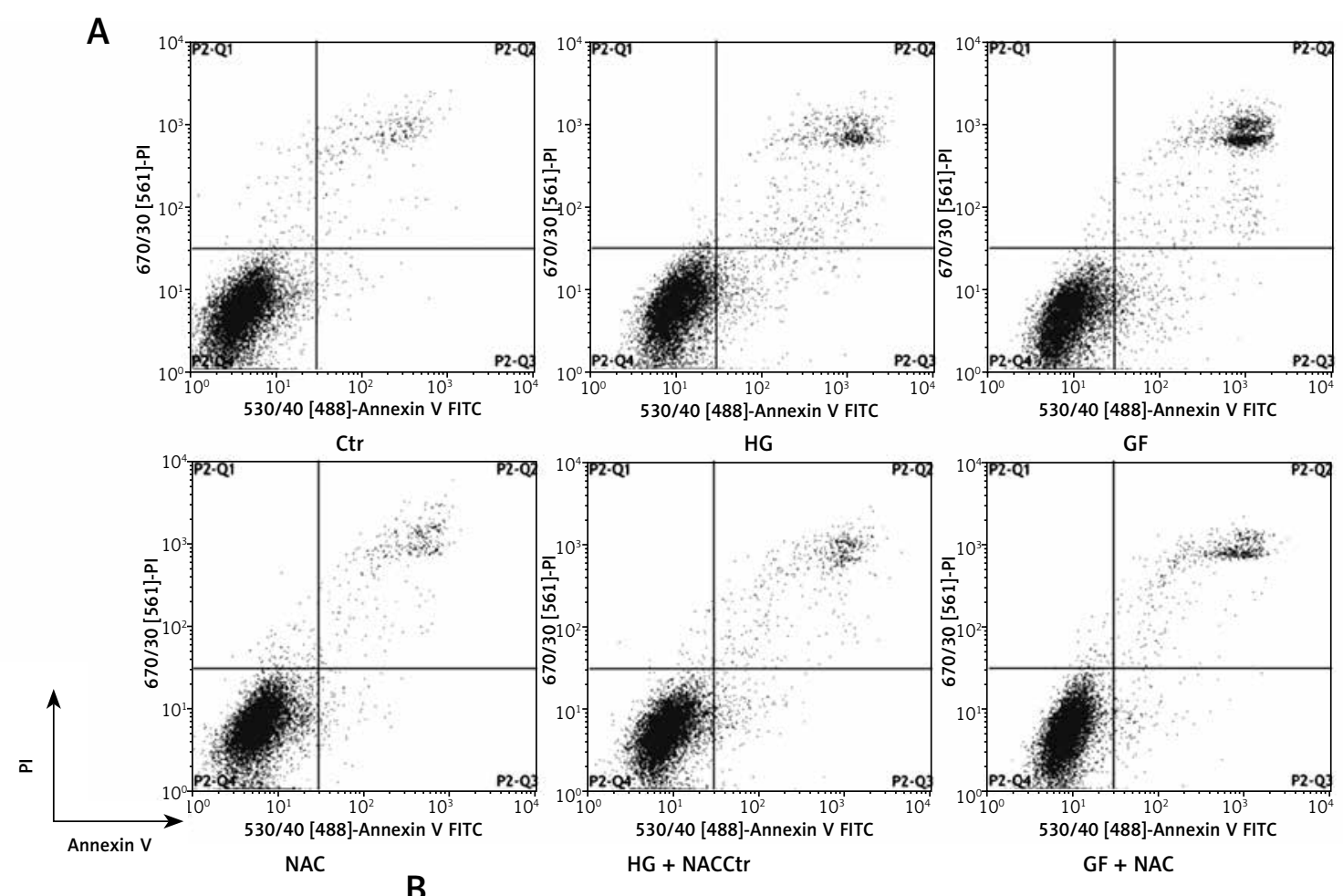

B
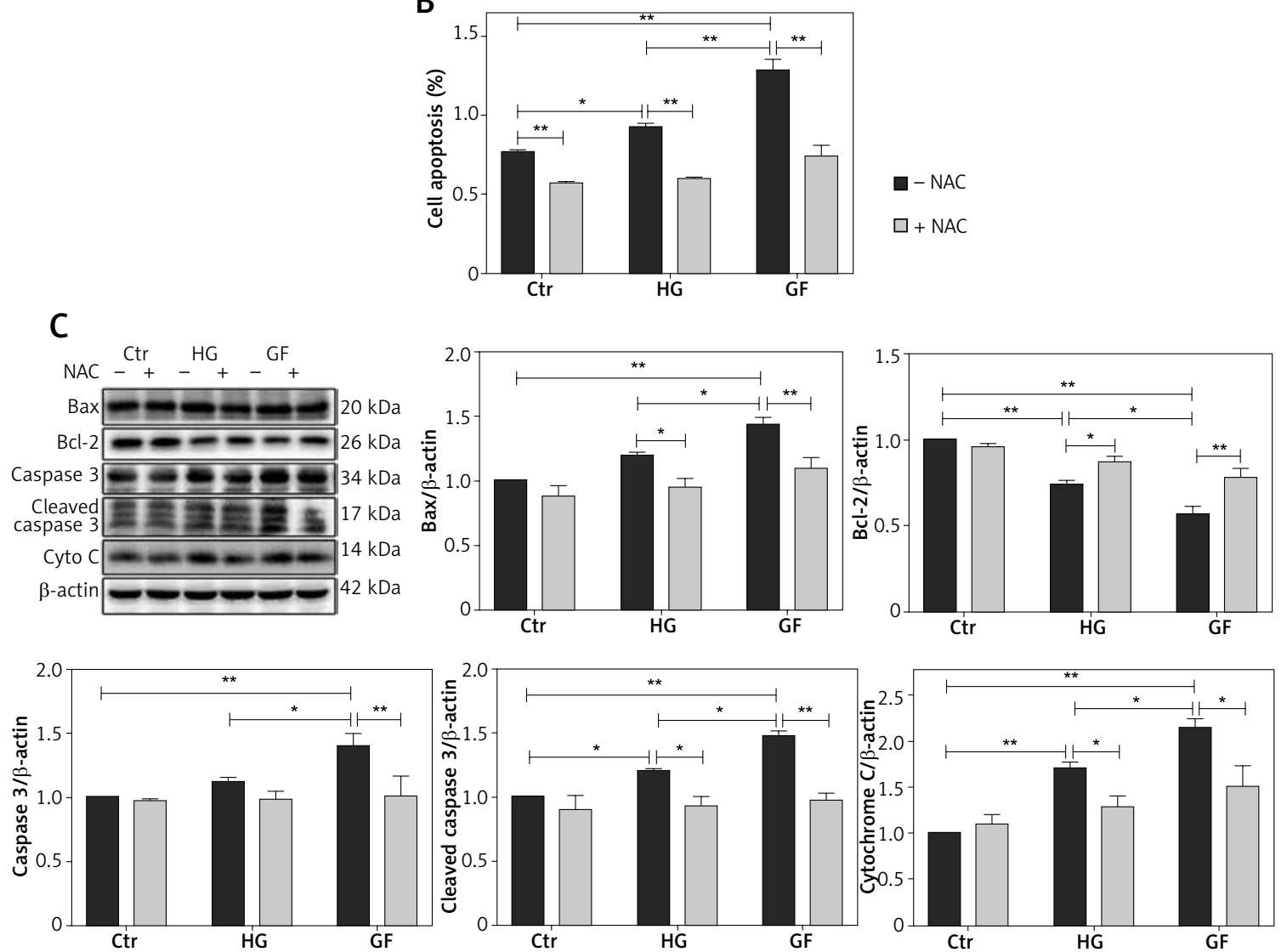

Figure 5. The role of oxidative stress in apoptosis of osteoblasts. A - Flow cytometry was used to test the apoptotic rates of osteoblasts after different treatments. B - Quantification of apoptotic rates induced by high glucose and glucose fluctuation w/wo the presence of NAC. C - Western blot analysis of apoptosis-related proteins induced by high glucose and glucose fluctuation w/wo the presence of NAC. Quantitative analysis of Bax, Bcl-2, caspase 3, cleaved caspase 3 and cytochrome c protein levels in the absence or presence of NAC. Two-way ANOVA was used to analyze the data. Independent experiments were performed three times. The data are expressed as the mean \pm SEM; $p<0.05, " * p<0.01$ 

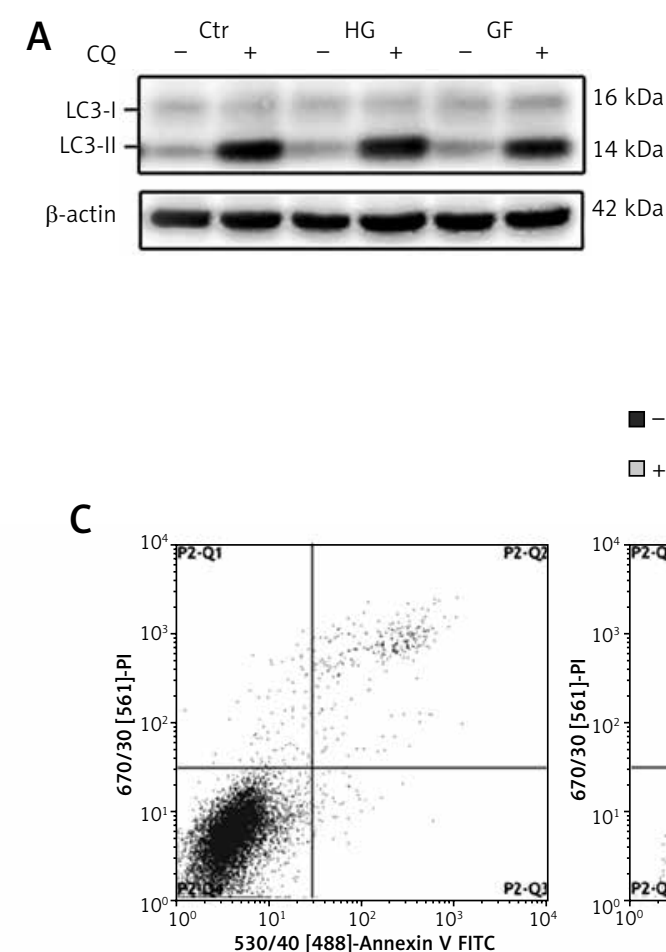

$\mathrm{Ctr}$

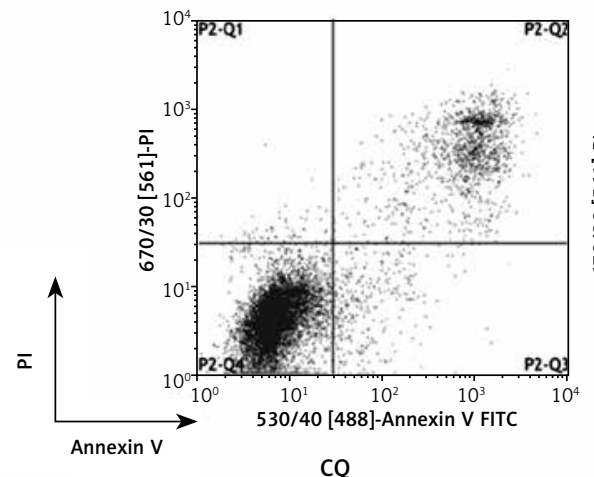

CQ

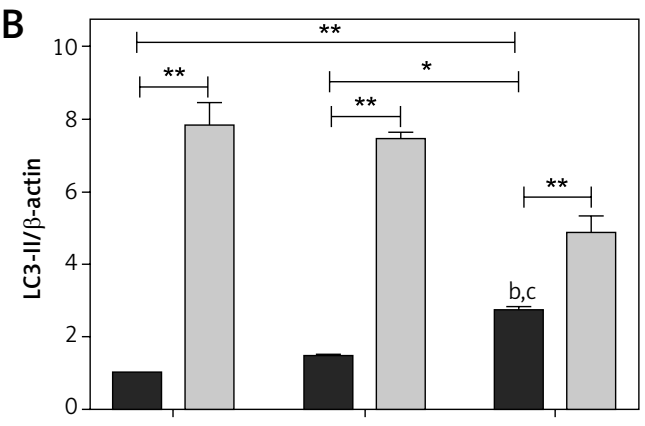

$\square+\mathrm{CQ}$

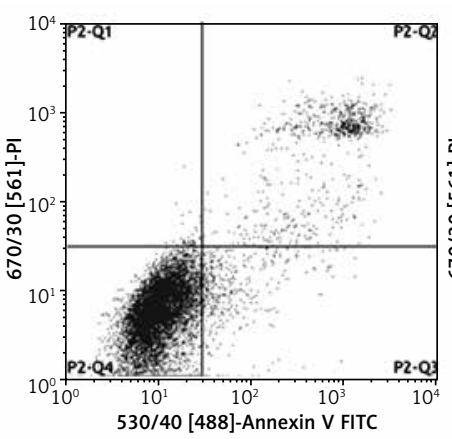

HG

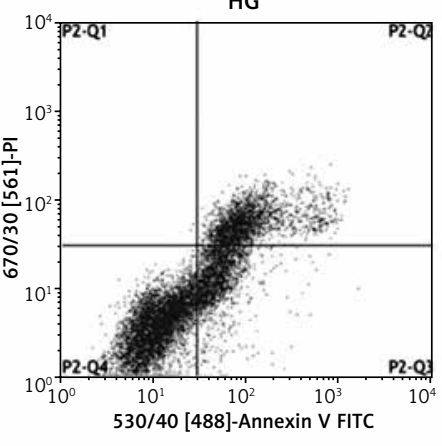

$\mathrm{HG}+\mathrm{CQ}$

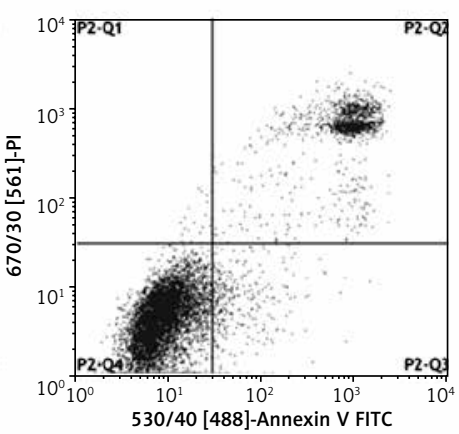

GF

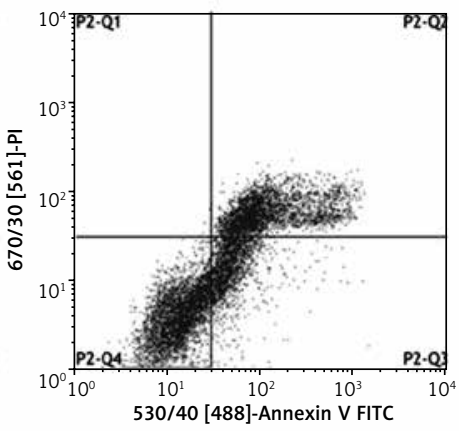

$\mathrm{GF}+\mathrm{CQ}$

D

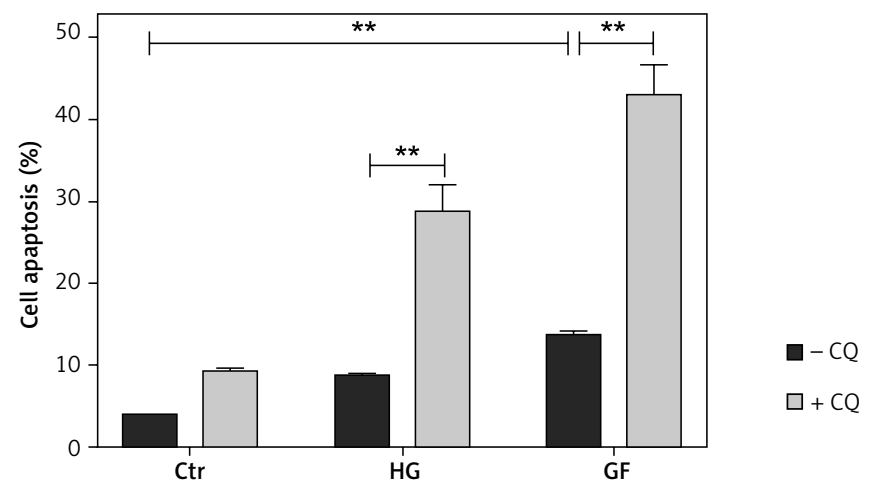

Figure 6. The role of autophagy in apoptosis of osteoblasts under different treatments. A - Western blot analysis of LC3-II protein expression induced by high glucose and glucose fluctuation w/wo the presence of CQ. B - Quantitative analysis of LC3-II protein levels in the absence or presence of CQ. C - Flow cytometry was used to evaluate the apoptosis of osteoblasts under different treatments. D - Quantification of apoptotic rates of osteoblasts with high glucose and glucose fluctuation treatment w/wo the presence of CQ. Two-way ANOVA was used to analyze the data. Independent experiments were performed three times. The data are expressed as the mean \pm SEM; ${ }^{*} p<0.05 ;{ }^{* *} p<0.01$ 
dative stress [39]. It was observed that high glucose induced the upregulation of an oxidative stress marker as well as an inflammation marker, which may further trigger apoptosis [41]. In vitro, oxidative stress has been reported to inhibit the differentiation of osteoblasts and induce the apoptosis of osteoblasts [46]. Meanwhile, the increase of intracellular oxidative stress was closely related to the occurrence and development of dysfunction of osteoblasts and osteoporosis [30, 4749]. ROS is the product of normal metabolism and is important for cell signaling and homeostasis. However, excessive ROS can lead to cell damage [50]. Meanwhile, mitochondria are another source of ROS production. Here, we treated MC3T3-E1 osteoblasts with high glucose $(25.0 \mathrm{mmol} / \mathrm{l})$ and glucose fluctuation $(5.5 / 25.0 \mathrm{mmol} / \mathrm{l})$. Our results showed that high glucose and glucose fluctuation treatments might increase the intracellular and mitochondrial ROS production in osteoblasts, especially after glucose fluctuation treatment (Figure $2 \mathrm{~A}$ ), which is in accordance with a previous study [40]. Also, glucose fluctuation caused a decrease of $\Delta \Psi \mathrm{m}$ and an increase of cytochrome $C$ protein expression (Figures $2 \mathrm{~B}$ and $5 \mathrm{C}$ ), indicating the damage of intracellular mitochondria. In addition, our results revealed an effect of proliferation inhibition and apoptosis initiation on osteoblasts after high glucose and glucose fluctuation treatment (Figure 1). Also, this effect was more pronounced in the GF group. Above all, the high glucose and glucose fluctuation could induce oxidative stress and damage of osteoblasts.

Autophagy is a basic and crucial physiological activity for cells to adapt to the changing microenvironment [11]. It plays a major role in determining cell fate and is involved in growth and development, cellular homeostasis, and physiological or pathological processes [51]. Hocking et al. found that the dysregulation of autophagy was associated with the pathogenesis of bone or cartilage diseases, such as osteoporosis, Paget bone disease and osteoarthritis [33]. We found that high glucose and glucose fluctuation resulted in significant up-regulation of expression of autophagy-associated proteins LC3-II and Beclin 1 as well as the expression of the mitochondrial autophagy-associated protein Bnip3, and down-regulation of p62 (Figure 3 B), which can bind with LC3 and promote degradation of polyubiquitinated protein aggregates, in MC3T3-E1 osteoblasts, especially in the GF group. Also, LC3-II was significantly increased after treatment with CQ (Figure $6 \mathrm{~A}$ ). CQ, as an autophagy inhibitor, can increase accumulation of LC3-II by raising the lysosomal $\mathrm{pH}$ or inhibiting lysosome-mediated proteolysis, by which the autophagosome-lysosome fusion step of autophagy would be interrupted [17]. Furthermore, LC3-II, as a receptor of the phagocytic membrane, plays an important role in the formation of autophagy [52], while Beclin1 is mainly involved in the initiation of autophagy. As a result, Beclin1 expression was not changed after adding CQ to block autophagosomal degradation (data not shown). TEM analyses also revealed that glucose fluctuation increased the number of autophagosomes (Figure 3 A). These results suggest that autophagy might be activated under the treatment of high glucose and glucose fluctuation, especially the latter.

An increased ROS level has been reported to affect the stability of cell function and was closely related to autophagy and apoptosis [26]. In the present study, glucose fluctuation led to a change of apoptosis-related protein expression, such as decreased $\mathrm{Bcl}-2$ expression as well as increased Bax, caspase 3 and cleaved caspase 3 expression (Figure $5 \mathrm{C}$ ). With the treatment of antioxidant NAC, ROS production was reduced (Figure $4 \mathrm{~A}$ ). Moreover, the increase of apoptosis and changes of protein expression induced by glucose fluctuation were reversed by NAC in MC3T3-E1 osteoblasts (Figure $5 \mathrm{C}$ ). Our study showed that NAC not only blocked the ROS production in the MC3T3-E1 osteoblasts, but also inhibited the apoptosis as well as their related protein expression. Then, NAC also suppressed the autophagy-related protein expression induced by glucose fluctuation (Figure 4 B), which indicated that autophagy was closely related to the ROS level in the osteoblasts. This might be related to the intracellular calcium overload induced by ROS [25, 53], which needs further verification. Taken together, these results reveal that ROS may play a role in the apoptosis and autophagy induced by high glucose and glucose fluctuation.

Autophagy plays an important role in the maintenance of cellular homeostasis. Here, we found that after treatment with CQ, an autophagy inhibitor, the apoptotic rates of osteoblasts were increased (Figure $6 \mathrm{C}$ ), which indicates that autophagy induced by high glucose and glucose fluctuation might protect MC3T3-E1 osteoblasts from apoptosis. In addition, there are various pathways that could influence autophagy, and we found that both $\mathrm{p}$-Akt/Akt and $\mathrm{p}-\mathrm{mTOR} / \mathrm{mTOR}$ were decreased in the GF group. In a previous study, Ying el al. found that glucose fluctuation increased mesangial cell apoptosis and that was related to the Akt pathway [44]. Also, other studies show that oxidative stress and autophagy are related to the Akt pathway [54, 55]. Byun et al. showed that Bnip3 affected autophagy by regulating the Akt-mTOR-p70S6K pathway [56]. However, our present study was not profound enough. Specially, more accurate methods are needed to detect the production of ROS and the intensity of fluores- 
cence images should be further quantified. Therefore, further studies are needed.

In conclusion, we found that high glucose and glucose fluctuation caused proliferation inhibition and induced apoptosis and autophagy in the MC3T3-E1 osteoblasts, and this effect was more profound under glucose fluctuation. The induction of autophagy and apoptosis was probably related to ROS production, which might be caused by damaged mitochondria. Additionally, further studies should be performed to investigate which organelles or molecules are involved in autophagy and how they regulate MC3T3-E1 osteoblasts under different glucose concentrations (high glucose and glucose fluctuation).

\section{Acknowledgments}

This study was supported by the Chongqing Medical Research Fund (grant number 2012-2041).

\section{Conflict of interest}

The authors declare no conflict of interest.

\section{References}

1. Chen P, Li Z, Hu Y. Prevalence of osteoporosis in China: a meta-analysis and systematic review. BMC Public Health 2016; 16: 1039.

2. Cho NH, Shaw JE, Karuranga S, et al. IDF Diabetes Atlas: Global estimates of diabetes prevalence for 2017 and projections for 2045. Diabetes Res Clin Pract 2018; 138: 271-81.

3. Looker AC, Sarafrazi Isfahani N, Fan B, Shepherd JA. Trends in osteoporosis and low bone mass in older US adults, 2005-2006 through 2013-2014. Osteoporos Int 2017; 28: 1979-88.

4. Noel SE, Mangano KM, Griffith JL, Wright NC, Dawson-Hughes B, Tucker KL. Prevalence of osteoporosis and low bone mass among puerto rican older adults. J Bone Miner Res 2018; 33: 396-403.

5. Wongdee K, Charoenphandhu N. Osteoporosis in diabetes mellitus: possible cellular and molecular mechanisms. World J Diabetes 2011; 2: 41-8.

6. Wongdee K, Charoenphandhu N. Update on type 2 diabetes-related osteoporosis. World J Diabetes 2015; 6: 673-8.

7. Oud L, Spellman C. The association of glucose variability and home discharge among survivors of critical Illness managed with a computerized decision-support tool for glycemic control. J Diabetes Sci Technol 2014; 8: 277-85.

8. Ouyang L, Shi Z, Zhao S, et al. Programmed cell death pathways in cancer: a review of apoptosis, autophagy and programmed necrosis. Cell Prolif 2012; 45: 487-98.

9. Levine B, Kroemer G. Autophagy in the pathogenesis of disease. Cell 2008; 132: 27-42.

10. Mizushima N, Levine B, Cuervo AM, Klionsky DJ. Autophagy fights disease through cellular self-digestion. Nature 2008; 451: 1069-75.

11. Maiuri MC, Kroemer G. Autophagy in stress and disease. Cell Death Differ 2015; 22: 365-6.
12. Song C, Song C, Tong F. Autophagy induction is a survival response against oxidative stress in bone marrow-derived mesenchymal stromal cells. Cytotherapy 2014; 16 : 1361-70.

13. Kang X, Yang W, Feng D, et al. Cartilage-specific autophagy deficiency promotes ER stress and impairs chondrogenesis in PERK-ATF4-CHOP-dependent manner. J Bone Miner Res 2017; 32: 2128-41.

14. Nollet M, Santucci-Darmanin S, Breuil V, et al. Autophagy in osteoblasts is involved in mineralization and bone homeostasis. Autophagy 2014; 10: 1965-77.

15. Piemontese M, Onal M, Xiong J, et al. Low bone mass and changes in the osteocyte network in mice lacking autophagy in the osteoblast lineage. Sci Rep 2016; 6: 24262.

16. Cao Y, Klionsky DJ. Physiological functions of Atg6/ Beclin 1: a unique autophagy-related protein. Cell Res 2007; 17: 839-49.

17. Klionsky DJ, Abdelmohsen K, Abe A, et al. Guidelines for the use and interpretation of assays for monitoring autophagy (3rd edition). Autophagy 2016; 12: 1-222.

18. Komatsu M, Wang QJ, Holstein GR, et al. Essential role for autophagy protein Atg7 in the maintenance of axonal homeostasis and the prevention of axonal degeneration. Proc Natl Acad Sci USA 2007; 104: 14489-94

19. Giacco F, Brownlee M. Oxidative stress and diabetic complications. Circ Res 2010; 107: 1058-70.

20. Kim MK, Jung HS, Yoon CS, et al. The effect of glucose fluctuation on apoptosis and function of INS-1 pancreatic beta cells. Korean Diabetes J 2010; 34: 47-54.

21. Sun LQ, Xue B, Li XJ, et al. Inhibitory effects of salvianolic acid $B$ on apoptosis of Schwann cells and its mechanism induced by intermittent high glucose. Life Sci 2012; 90: 99-108.

22. Jiao H, Xiao E, Graves DT. Diabetes and its effect on bone and fracture healing. Curr Osteoporos Rep 2015; 13: 327-35.

23. Paneni F, Beckman JA, Creager MA, Cosentino F. Diabetes and vascular disease: pathophysiology, clinical consequences, and medical therapy: part I. Eur Heart J 2013; 34: 2436-43.

24. Tanaka S, Aida K, Nishida Y, Kobayashi T. Pathophysiological mechanisms involving aggressive islet cell destruction in fulminant type 1 diabetes. Endocr J 2013; 60: 837-45.

25. Wang X, Feng Z, Li J, Chen L, Tang W. High glucose induces autophagy of MC3T3-E1 cells via ROS-AKT-mTOR axis. Mol Cell Endocrinol 2016; 429: 62-72.

26. Li L, Tan J, Miao Y, Lei P, Zhang Q. ROS and autophagy: interactions and molecular regulatory mechanisms. Cell Mol Neurobiol 2015; 35: 615-21.

27. Chakrabarti S, Jahandideh F, Wu J. Food-derived bioactive peptides on inflammation and oxidative stress. Biomed Res Int 2014; 2014: 608979.

28. Fandy TE, Jiemjit A, Thakar M, Rhoden P, Suarez L, Gore $\mathrm{SD}$. Decitabine induces delayed reactive oxygen species (ROS) accumulation in leukemia cells and induces the expression of ROS generating enzymes. Clin Cancer Res 2014; 20: 1249-58.

29. Kaminskyy VO, Boris Z. Free radicals in cross talk between autophagy and apoptosis. Antioxid Redox Signal 2014; 21: 86-102.

30. Tang ZH, Cao WX, Su MX, Chen X, Lu JJ. Osimertinib induces autophagy and apoptosis via reactive oxygen species generation in non-small cell lung cancer cells. Toxicol Appl Pharmacol 2017; 321: 18-26. 
31. Booth LA, Seyedmehrad T, Hamed HA, Nichola C, Paul D. The role of cell signalling in the crosstalk between autophagy and apoptosis. Cell Signal 2014; 26: 549-55.

32. Perez MJ, Quintanilla RA. Development or disease: duality of the mitochondrial permeability transition pore. Dev Biol 2017; 426: 1-7.

33. Hocking LJ, Whitehouse C, Helfrich MH. Autophagy: a new player in skeletal maintenance? J Bone Miner Res 2012; 27: 1439-47.

34. Janghorbani M, Van Dam RM, Willett WC, Hu FB. Systematic review of type 1 and type 2 diabetes mellitus and risk of fracture. Am J Epidemiol 2007; 166 495-505.

35. Johnell O, Kanis JA. An estimate of the worldwide prevalence and disability associated with osteoporotic fractures. Osteoporos Int 2006; 17: 1726-33.

36. Ducy P, Schinke T, Karsenty G. The osteoblast: a sophisticated fibroblast under central surveillance. Science 2000; 289: 1501-4.

37. Masse PG, Pacifique MB, Tranchant CC, et al. Bone metabolic abnormalities associated with well-controlled type 1 diabetes (IDDM) in young adult women: a disease complication often ignored or neglected. J Am Coll Nutr 2010; 29: 419-29.

38. Meng X, Gong C, Cao B, et al. Glucose fluctuations in association with oxidative stress among children with T1DM: comparison of different phases. J Clin Endocrinol Metab 2015; 100: 1828-36.

39. Monnier L, Mas E, Ginet C, et al. Activation of oxidative stress by acute glucose fluctuations compared with sustained chronic hyperglycemia in patients with type 2 diabetes. JAMA 2006; 295: 1681-7.

40. Wu N, Shen H, Liu H, Wang Y, Bai Y, Han P. Acute blood glucose fluctuation enhances rat aorta endothelial cell apoptosis, oxidative stress and pro-inflammatory cytokine expression in vivo. Cardiovasc Diabetol 2016; 15: 109.

41. Wu N, Shen H, Wang Y, et al. Role of the PKCbetall/JNK signaling pathway in acute glucose fluctuation-induced apoptosis of rat vascular endothelial cells. Acta Diabetol 2017; 54: 727-36.

42. Xia J, Qu Y, Yin C, Xu D. Optical coherence tomography assessment of glucose fluctuation impact on the neoin timal proliferation after stent implantation in a diabetic/hypercholesterolemic swine model. Int Heart J 2017; 58: 608-14.

43. Yamagishi S. Role of advanced glycation end products (AGEs) in osteoporosis in diabetes. Curr Drug Targets 2011; 12: 2096-102.

44. Ying C, Wang S, Lu Y, et al. Glucose fluctuation increased mesangial cell apoptosis related to AKT signal pathway. Arch Med Sci 2019; 15: 730-7.

45. Ceriello A, Esposito K, Piconi L, et al. Oscillating glucose is more deleterious to endothelial function and oxidative stress than mean glucose in normal and type 2 diabetic patients. Diabetes 2008; 57: 1349-54.

46. Fatokun AA, Stone TW, Smith RA. Hydrogen peroxide-induced oxidative stress in MC3T3-E1 cells: the effects of glutamate and protection by purines. Bone 2006; 39: 542-51.

47. Dan L, Liangbi X, Maowei Y, et al. ZnT7 can protect MC3T3-E1 cells from oxidative stress-induced apoptosis via PI3K/Akt and MAPK/ERK signaling pathways. Cell Signal 2013; 25: 1126-35.

48. Feng YL, Tang XL. Effect of glucocorticoid-induced oxidative stress on the expression of Cbfa1. Chem Biol Interact 2014; 207: 26-31.
49. Wauquier F, Leotoing L, Coxam V, Guicheux J, Wittrant Y. Oxidative stress in bone remodelling and disease. Trends Mol Med 2009; 15: 468-77.

50. Circu ML, Aw TY. Reactive oxygen species, cellular redox systems, and apoptosis. Free Radic Biol Med 2010; 48 749-62.

51. Eisenberg-Lerner A, Bialik S, Simon HU, Kimchi A. Life and death partners: apoptosis, autophagy and the cross-talk between them. Cell Death Differ 2009; 16: 966-75

52. Mukhopadhyay S, Panda PK, Sinha N, Das DN, Bhutia SK. Autophagy and apoptosis: where do they meet? Apoptosis 2014; 19: 555-66.

53. Scherz-Shouval R, Elazar Z. Regulation of autophagy by ROS: physiology and pathology. Trends Biochem Sci 2011; 36: 30-8.

54. Shi X, Guan Y, Jiang S, Li T, Sun B, Cheng H. Renin-angiotensin system inhibitor attenuates oxidative stress induced human coronary artery endothelial cell dys function via the PI3K/AKT/mTOR pathway. Arch Med Sci 2019; 15: 152-64.

55. Guo H, He Y, Bu C, Peng Z. Antitumor and apoptotic effects of 5-methoxypsoralen in U87MG human glioma cells and its effect on cell cycle, autophagy and PI3K/ Akt signaling pathway. Arch Med Sci 2019; 15: 1530-8.

56. Byun YJ, Kim SK, Kim YM, Chae GT, Jeong SW, Lee SB. Hydrogen peroxide induces autophagic cell death in C6 glioma cells via BNIP3-mediated suppression of the mTOR pathway. Neurosci Lett 2009; 461: 131-5. 